Pacific Journal of Mathematics

PERMANENTS OF CYCLIC MATRICES 


\title{
PERMANENTS OF CYCLIC MATRICES
}

\author{
M. F. Tinsley
}

1. Introduction. Let $A=\left[a_{i j}\right]$ be an $n \times n$ matrix with non-negative real entries. The permanent of $A$, written $P(A)$, is defined by

$$
P(A)=\sum a_{1 i_{1}} a_{2 i_{2}} \cdots a_{n i_{n}},
$$

where the summation extends over the $n$ ! permutations of the integers $i_{1}, i_{2}, \cdots, i_{n}$. Thus the permanent and determinant are alike in definition except for sign changes. However unlike the determinant, the properties of the permanent function are little understood. The object of this paper is to determine for a certain class of matrices those matrices $A$ for which the permanent and determinant are equal in absolute value. This property we write $P(A)=|D(A)|$. For such matrices the permanent may then be evaluated by the determinant.

Let $A=\left[a_{i j}\right]$ be an $n \times n$ matrix composed of 0 's and 1 's with row and column sums equal to $s$. Let $\Sigma=\left[\sigma_{i j}\right]$ be a permutation submatrix of $A$. This means that $\Sigma$ is a permutation matrix of order $n$ such that $\sigma_{k l}=1$ implies $a_{k l}=1$. With $\Sigma$ we associate a permutation $\Sigma^{\prime}$ of the letters $1,2, \cdots, n$

$$
\Sigma^{\prime}(i)=j \text { if and only if } \sigma_{i j}=1 .
$$

It follows by definition then that $P(A)=|D(A)|$ if and only if every $\Sigma^{\prime}$ is even or else every $\Sigma^{\prime}$ is odd.

By a theorem due to König (1), the matrix $A$ may be written as a sum of $s$ permutation matrices,

$$
A=\pi_{1}+\pi_{2}+\cdots+\pi_{s} .
$$

For convenience we will say that $A$ is defined by the $s$ permutations $\pi_{1}^{\prime}, \pi_{2}^{\prime}, \cdots, \pi_{s}^{\prime}$. If $\pi_{k}^{\prime} \pi_{j}^{\prime}=\pi_{j}^{1} \pi_{k}^{\prime}$ for each $j$ and $k$, then $A$ will be called abelian. If for $i=1,2, \cdots, s, \pi_{i}^{\prime}=(1,2, \cdots, n)^{a_{i}}$ where $0 \leqq d_{i}<n$, then $A$ is cyclic and will be said to be defined by the difference $d_{1}, d_{2}, \cdots, d_{s}$ $\bmod n$.

Now let $C$ be the $7 \times 7$ cyclic matrix defined by the differences $0,1,3, \bmod 7$. The main result of the paper may be stated as follows:

Let $A$ be an $n \times n$ abelian matrix with $s \geqq 3$ ones in each row and column. Then $P(A)=|D(A)|$ if and only if $s=3, n=7 e$ and upon permutations of rows and columns $A$ is transformed into the direct sum of $C$ taken e times.

Received September 6, 1958, and in revised form, August 24, 1959. This research was supported in part by the Office of Ordnance Research. 
Most of the results of this paper are taken from the author's doctoral thesis written at the Ohio State University under the supervision of Prof. H. J. Ryser. Theorem 1, 2, 3 and 6 are from that source, the proof of Theorem 6 being essentially altered here.

2. Representation of Cycles. Let $A$ be a 0,1 matrix of order $n$ with $s$ ones in each row and column. By (1.3) we may write $A=\pi_{1}+$ $\pi_{2}+\cdots+\pi_{s}$. The matrix $B=\pi_{s}^{-1} A$ has ones on the main diagonal and possesses the same permanent and, apart from sign, the same determinant as $A$. Thus there is no loss in supposing $A$ has ones on the main diagonal. If now $\Sigma$ is a permutation submatrix of $A$ any cycle of $\Sigma^{\prime}$ also corresponds to a permutation submatrix of $A$. Hence $P(A)=|D(A)|$ if and only if all such cycles are even. We shall refer to these cycles as the cycles of $A$.

If $P(A)=|D(A)|$ and $B=A-\pi_{s}$ then $B$ has the same property since any term in the expansion of $D(B)$ also contributes to $D(A)$. Thus any results for matrices $A$ with $P(A)=|D(A)|$ and $s=3$ will also apply to matrices with $t \geqq 3$ ones in each row and column. It develops, at east for the class of abelian matrices, that the analysis for $s=3$ is all lthat is necessary.

For the remainder of $\S \S 2,3$ and 4 only cyclic matrices will be considered. Let $A$ be cyclic of order $n$ and defined by the differences $0, d_{1}$ and $d_{2} \bmod n$. Reading $\bmod n$, a cycle of $A$ must have the form

$$
a \longrightarrow a+d_{i_{1}} \longrightarrow a+d_{i_{1}}+d_{i_{2}} \longrightarrow \cdots \longrightarrow a+d_{i_{1}}+d_{i_{2}}+\cdots+d_{i_{\mathfrak{j}}} \text {. }
$$

Here the $d_{i_{k}}$ 's are $d_{1}$ or $d_{2}$ and $d_{i_{1}}+d_{i_{2}}+\cdots+d_{i_{j}} \equiv 0(\bmod n)$. Now arrange the $d_{i_{k}}$ 's in a circle as follows:

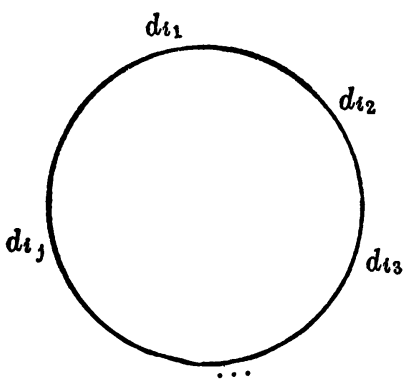

Then no consecutive selection of $t$ of the $d_{i_{k}}$ 's, $0<t<j$, has a sum divisible by $n$. Otherwise there would be a cycle within a cycle.

Conversely, let $d_{i_{1}}, d_{i_{2}}, \cdots, d_{i_{j}}$ be a sequence formed from $d_{1}$ and $d_{2}$ such that $d_{i_{1}}+d_{i_{2}}+\cdots+d_{i_{j}} \equiv 0(\bmod n)$ and, when arranged in a circle, no proper consecutive selection of the $d_{i_{k}}$ 's has a sum divisible by $n$. Then for each $b=1,2, \cdots, n$,

$$
b \longrightarrow b+d_{i_{1}}^{\prime} \longrightarrow b+d_{i_{1}}+d_{i_{2}} \longrightarrow \cdots \longrightarrow b+d_{i_{1}}+d_{i_{2}}+\cdots+d_{i_{j}}
$$


is a cycle of $A$.

Now for a cycle $a \rightarrow a+d_{i_{1}} \rightarrow a+d_{i_{i}}+d_{i_{j}} \rightarrow \cdots \rightarrow a+d_{i_{1}}+\cdots+d_{i_{j}}$, let $x_{k}$ denote the number of times that $d_{k}$ occurs among $d_{i_{1}}, d_{i_{2}}, \cdots, d_{i_{j}}$. Then $j=x_{1}+x_{2}$ and

$$
d_{1} x_{1}+d_{2} x_{2} \equiv 0(\bmod n) .
$$

Definition. We say that the solution $\left(x_{1}, x_{2}\right)$ of the congruence $d_{1} x+d_{2} y \equiv 0(\bmod n)$ represents a cycle of the matrix $A$. More precisely, let $d_{1} x^{\prime}+d_{2} y^{\prime} \equiv 0(\bmod n)$, where $0 \leqq x^{\prime}, y^{\prime}$ and $0<x^{\prime}+y^{\prime}$. Suppose there exists some arrangement of $x^{\prime} \alpha$ 's and $y^{\prime} \beta^{\prime}$ s in a circle with the following property; For all other solutions $x^{*}, y^{*}$ of (2.1) such that $0 \leqq x^{*} \leqq x^{\prime}, 0 \leqq y^{*} \leqq y^{\prime}$ and $0<x^{*}+y^{*}$, no consecutive selection of $x^{*}+y^{*} \alpha^{\prime}$ s and $\beta^{\prime}$ 's totals exactly $x^{*} \alpha$ 's. Then the solution $\left(x^{\prime}, y^{\prime}\right)$ represents a cycle. If no such arrangement exists $\left(x^{\prime}, y^{\prime}\right)$ does not represent a cycle.

Note that if $\left(y_{1}, y_{2}\right)$ represents a cycle then the cycle has length $y_{1}+y_{2}$ and hence is even or odd according as $y_{1}+y_{2}$ is odd or even. Thus to determine if $P(A)=|D(A)|$ it suffices to study the solutions of (2.1).

ExAmple 1. The $7 \times 7$ matrix $C$ defined by the differences $0,1,3$ mod 7 has permanent equal to determinant. For consider the solutions of $x+3 y \equiv 0(\bmod 7):(4,1),(1,2),(5,3),(2,4),(6,5),(3,6),(0,7),(7,0)$. One readily shows that $(2,4)$ can not represent a cycle and that only $(4,1),(1,2),(0,7)$ and $(7,0)$ may. Since the sums $4+1,1+2,0+7$ are odd it follows that $P(C)=|D(C)|$. Similarly one shows that the $7 \times 7$ matrix defined by the differences $0,1,5 \bmod 7$ has permanent equal to determinant.

3. Primitive Solutions. In this section we study a general congruence

$$
a x+b y \equiv 0(\bmod n),
$$

where $a$ and $b$ are positive integers not necessarily distinct and $x, y$ are non-negative integers.

Let $\left(x_{1}, y_{1}\right)$ and $\left(x_{2}, y_{2}\right)$ be solutions of (3.1). We write $\left(x_{1}, y_{1}\right) \geqq\left(x_{2}, y_{2}\right)$ provided $x_{1} \geqq x_{2}, y_{1} \geqq y_{2}$ and $\left(x_{1}, y_{1}\right)=\left(x_{2}, y_{2}\right)$ if $x_{1}=x_{2}$ and $y_{1}=y_{2}$. Furthermore, we write $\left(x_{1}, y_{1}\right)>\left(x_{2}, y_{2}\right)$ provided $x_{1}>x_{2}, y_{1} \geqq y_{2}$ or $x_{1} \geqq x_{2}, y_{1}>y_{2}$.

Now let $\left(x_{0}, y_{0}\right)$ be a solution of (3.1) such that both $x_{0}$ and $y_{0}$ are positive.

Definition. $\left(x_{0}, y_{0}\right)$ will be called primitive if for every solution 
$\left(x_{0}^{\prime}, y_{0}^{\prime}\right)$ such that $(0,0) \leqq\left(x_{0}^{\prime}, y_{0}^{\prime}\right) \leqq\left(x_{0}, y_{0}\right)$ either $\left(x_{0}^{\prime}, y_{0}^{\prime}\right)=(0,0)$ or $\left(x_{0}^{\prime}, y_{0}^{\prime}\right)=\left(x_{0}, y_{0}\right)$.

Suppose now that the $n \times n$ matrix $A$ is defined by the three differences $0, d_{1}, d_{2} \bmod n$. From the definition and the discussion of $\S 2$ it follows that primitive solutions of the congruence $d_{1} x+d_{2} y \equiv 0(\bmod n)$ must represent cycles of $A$. Thus the study of primitive solutions is suggested as a starting point in our investigations. The first theorem concerns the determination of the primitive solutions of (3.1).

Let $n \equiv n_{1}(\bmod d)$ and $d \equiv d_{1}\left(\bmod n_{1}\right)$, where $0 \leqq d_{1}<n_{1}<d<n$. To simplify the notation we shall set $F=n / d$ and $G=d / n_{1}$.

THEOREM 1. If the primitive solutions of $x^{\prime}+d_{1} y^{\prime} \equiv 0\left(\bmod n_{1}\right)$ are those solutions for which $y^{\prime}=i, j, k, \cdots$, then the primitive solutions of $x+d y \equiv 0(\bmod n)$ are those solutions for which

$$
y=1,2, \cdots,[F],[([i G]+1) F],[([j G]+1) F],[([k G]+1) F], \cdots .
$$

If $n \equiv 0(\bmod d)$, then the primitive solutions of $x+d y \equiv 0(\bmod n)$ are those solutions with $y=1,2, \cdots,[F]-1$.

Proof. Clearly the solutions of $x+d y \equiv 0(\bmod n)$ with $y=1,2, \cdots$, $[F]-1$ are primitive, since as $y$ increases the corresponding $x$ decreases. If $n$ is divisible by $d$ then these are all the primitive solutions. If $n \not \equiv 0(\bmod d)$ then the solution with $y=[F]$ is also primitive. If, in addition, $d_{1}=0$ then $n_{1}$ is the greatest common divisor of $n$ and $d$, and $\left(n_{1},[F]\right)$ is a primitive solution of $x+d y \equiv 0(\bmod n)$. Moreover, if $\left(x_{0}, y_{0}\right)$ is another solution and $y_{0}>[F]$, then $\left(x_{0}, y_{0}\right)$ is not primitive since $x_{0}$ must be a multiple of $n_{1}$. Thus in proving the theorem, both $n_{1}$ and $d_{1}$ may be supposed not zero.

Assume now that $[F]<v \neq[i F], i=1,2, \cdots, d-1$. Let $\left(x_{1}, y_{1}\right)$ be the solution of $x+d y \equiv 0(\bmod n)$ with $y_{1}=v$. Then there is a solution $\left(x^{\prime}, y^{\prime}\right)$ where $(0,0)<\left(x^{\prime}, y^{\prime}\right)<\left(x_{1}, y_{1}\right)$ and $y^{\prime}=[j F]$ for some $j, 1 \leqq j \leqq d-1$. To show this let $j$ be such that $[j F]<v<[(j+1) F]$. Since $[F] \leqq[(j+1) F]-[j F] \leqq[F]+1$, we have $v=[j F]+r, r \leqq[F]$. Then $x_{1}=(j+1) n-([j F]+r) d$. Now the $x^{\prime}$ corresponding to $y^{\prime}=[j F]$ is $j n-[j F] d$. Thus if we set $x^{\prime}=j n-[j F] d$ and $y^{\prime}=[j F]$, then

$x_{1}-x^{\prime}=\{(j+1) n-([j F]+r) d\}-\{j n-[j F]\}=n-r d \geqq n-[F] d>0$

and $\left(x^{\prime}, y^{\prime}\right)<\left(x_{1}, y_{1}\right)$. As a consequence, to determine the primitive solutions of the congruence $x+d y \equiv 0(\bmod n)$ it suffices to consider those solutions with $y=[i F], i=1,2, \cdots, d-1$.

LEMMA 1. If $0 \leqq a_{i}<d<n(i=1,2, \cdots, d-1)$ then

(1) in $\equiv a_{i}(\bmod d)$ if and only if $a_{i}+d\left[i F^{\prime}\right] \equiv 0(\bmod n)$. For 
$0 \leqq a_{i}<d<n$ and in $\equiv a_{i}(\bmod d)$

$(i=1,2, \cdots, d-1)$,

(2) $x_{0}=a_{g}, y_{0}=[g F]$ is a primitive solution of $x+d y \equiv 0(\bmod n)$ if and only if $a_{1}, a_{2}, \cdots, a_{g-1}$ are all $>a_{g}>0$.

Proof. (1) Necessity If in $\equiv a_{i}(\bmod d)$ then in $=a_{i}+u_{i} d$ for some $u_{i}$, and $u_{i}=\left(i n-a_{i}\right) / d=[i F]$. Substituting $[i F]$ for $u_{i}, a_{i}+d[i F]=$ in $\equiv 0(\bmod n)$.

Sufficiency. If $a_{i}+d[i F] \equiv 0(\bmod n)$ then since $i n \geqq d[i F]>(i-1) n$, we have $a_{i}+d[i F]=i n$. Thus $i n \equiv a_{i}(\bmod d)$.

(2) Necessity. Let $x_{0}=a_{g}, y_{0}=[g F]$ be a primitive solution of $x+d y \equiv 0(\bmod n)$ and $x^{\prime}, y^{\prime}$ another solution. If $0<y^{\prime}<y_{0}$, then we must have $x^{\prime}>x_{0}$. Thus for $y^{\prime}=[j F](j=1,2, \cdots, g-1), a_{j}=x^{\prime}>x_{0}=a_{g}$.

Sufficiency. If $\left(x_{0}, y_{0}\right)$ is not primitive then there is a solution $\left(x_{1}, y_{1}\right)$ such that $(0,0)<\left(x_{1}, y_{1}\right)<\left(x_{0}, y_{0}\right)$. If now $y_{1} \geqq[F]$, then by the earlier remarks of this section there is a solution $\left(x^{\prime}, y^{\prime}\right)$ such that $(0,0)<\left(x^{\prime}, y^{\prime}\right) \leqq\left(x_{1}, y_{1}\right)$ and $y^{\prime}=[j F]$ for some $j, 1 \leqq j \leqq d-1$. Since $y^{\prime}<y_{0}$ we have $j<g$. But also since $x^{\prime} \leqq x_{0}$, we have $a_{j} \leqq a_{g}$. If $y_{1}<[F]$, then $a_{1}<x_{1}$ so $a_{1}<x_{0}=a_{g}$ and $1<g$. In either case we contradict $a_{1}, a_{2}, \cdots, a_{g_{-1}}>a_{g}$.

Now consider the following table of values defined for each $k=$ $0,1,2, \cdots, n_{1}-1$.

\begin{tabular}{cc}
$b_{i}$ & $i$ \\
\hline$([k G]+1) n_{1}-k d$ & {$[k G]+1$} \\
$([k G]+2) n_{1}-k d$ & {$[k G]+2$} \\
$\vdots$ & $\vdots$ \\
{$[(k+1) G] n_{1}-k d$} & {$[(k+1) G]$}
\end{tabular}

It follows readily that for the $a_{i}$ of Lemma $1, a_{i} \equiv i n \equiv i n_{1} \equiv b_{i}(\bmod d)$, that $0<b_{i} \leqq d$ and that $b_{i}$ increases with $i$. Here $i$ is understood as limited to those values in the table. Now $(k+1) d \geqq[(k+1) G] n_{1}$ implies $[(k+1) G] n_{1}-k d \leqq d$ where equality holds only if $(k+1) d \equiv 0\left(\operatorname{mon} n_{1}\right)$. Thus $b_{i}=a_{i}$ unless $i=[(k+1) G]$ and $(k+1) d \equiv 0\left(\bmod n_{1}\right)$. In this case $a_{i}=0$ and $b_{i}=d$.

Now let $\left(a_{j},[j F]\right)$ be a primitive solution of the congruence $x+d y \equiv 0(\bmod n)$. The integer $j$ must occur as some $i$ in table (4.1). Since $b_{i}$ increses with $i$, we must have $j=[k G]+1$, for some $k, 0 \leqq$ $k \leqq n_{1}-1$.

Finally, $k d \equiv 0(\bmod n)$ implies $k$ is divisible by $u=n_{1} /(d, n)$. Thus since $(0,[u G F])$ is a solution, those solutions $\left(a_{i},[i F]\right)$, where $i=[k G]+1$ and $k d \equiv 0\left(\bmod n_{1}\right)$, are not primitive.

Next consider the solution of $x^{\prime}+d_{1} y^{\prime} \equiv 0\left(\bmod n_{1}\right)$. For $k=$ 
$1,2, \cdots, n_{1}-1$ define $\alpha_{k} \equiv-k d \equiv-k d_{1}\left(\bmod n_{1}\right)$ where $0 \leqq \alpha_{k}<n_{1}$. Then the nontrivial solutions are $\left(\alpha_{1}, 1\right),\left(\alpha_{2}, 2\right), \cdots,\left(\alpha_{n_{1}-1}, n_{1}-1\right)$.

LEMma 2. Let $1 \leqq k, l<n$ where $k d$ and $l d$ are not divisible by $n_{1}$. If $e=[k G]+1$ and $f=[l G]+1$, then

$$
a_{e}-a_{f}=\alpha_{k}-\alpha_{l} \text {. }
$$

Proof. $\quad a_{e}=b_{e}=e n_{1}-k d$ and $a_{f}=b_{f}=f n_{1}-l d$ while

$$
\alpha_{k}=n_{1}-\left\{k d-[k G] n_{1}\right\}
$$

and

$$
\alpha_{\imath}=n_{1}-\left\{l d-[l G] n_{1}\right\} .
$$

Thus $a_{e}-a_{f}=([k G]-[l G]) n_{1}+(l-k) d=\alpha_{k}-\alpha_{l}$.

Now we may prove the theorem. List the primitive solutions of $x+d y \equiv 0(\bmod n)$ as $(n-d, 1),(n-2 d, 2), \cdots,\left(n_{1},[F]\right)=\left(a_{1},[F]\right)$, $\left(a_{j_{1}},\left[j_{1} F\right]\right),\left(a_{j_{2}},\left[j_{2} F\right]\right), \cdots,\left(a_{j_{v}},\left[j_{v} F\right]\right)$, where by primitivity $a_{1}>a_{j_{1}}>a_{j_{2}}>$ $\cdots>a_{v_{v}}>0$ and $1<j_{1}<j_{2}<\cdots<j_{v}$. We have seen that each $j_{t}$ must have the form $[k G]+1$ where $k d \not \equiv 0\left(\bmod n_{1}\right)$. For $1 \leqq p \leqq v$ define $k_{p}$ by setting $j_{p}=\left[k_{p} G\right]+1$ and consider the following solutions of $x^{\prime}+d_{1} y^{\prime} \equiv 0\left(\bmod n_{1}\right)$ :

$$
\left(\alpha_{k_{1}}, k_{1}\right),\left(\alpha_{\kappa_{2}}, k_{2}\right), \cdots,\left(\alpha_{\kappa_{v}}, k_{v}\right) .
$$

By Lemma 2, $\alpha_{\kappa 1}>\alpha_{\kappa_{2}}>\cdots>\alpha_{\kappa_{v}}$. We cannot have $\alpha_{\kappa_{v}}=0$ for then $k_{v} d \equiv 0\left(\bmod n_{1}\right)$.

If for some $u, 1 \leqq u \leqq v,\left(\alpha_{\kappa_{u}}, k_{u}\right)$ is not a primitive solution of $x^{\prime}+d_{1} y^{\prime} \equiv 0\left(\bmod n_{1}\right)$, then there is a solution $\left(\alpha_{r}, r\right)$ such that $(0,0)<\left(\alpha_{r}, r\right)<\left(\alpha_{\kappa_{u}}, k_{u}\right)$. Set $H=1 /(d, n)=1 /\left(d_{1}, n_{1}\right)$. If $\alpha_{r}=0$ then $r d_{1} \equiv 0\left(\bmod n_{1}\right)$ and $k_{u}>n_{1} H$.

But then $j_{u} \geqq\left[n_{1} H G\right]+1=d H+1$ and $(0,[d H F])<\left(a_{j_{u}},\left[j_{u} F\right]\right)$, contradicting the primitivity of $\left(a_{j_{u}},\left[j_{u} F\right]\right)$.

If $\alpha_{r}>0$ then by Lemma 2 , if $f=[r G]+1,(0,0)<\left(a_{f},[f F]\right)<\left(a_{j_{u}},\left[j_{u} F\right]\right)$ again contradicting the primitivity of $\left(a_{j_{u}},\left[j_{u} F\right]\right)$. Thus for $1 \leqq p \leqq v$, $\left(\alpha_{\kappa_{p}}, k_{p}\right)$ is a primitive solution of $x^{\prime}+d_{1} y^{\prime} \equiv 0\left(\bmod n_{1}\right)$. To complete the proof of the theorem we must show that there are no others.

Suppose $\left(\alpha_{q}, q\right)$ is a primitive solution of $x^{\prime}+d_{1} y^{\prime} \equiv 0\left(\bmod n_{1}\right)$. Since $\alpha_{1}, \alpha_{2}, \cdots, \alpha_{q-1}$ are $>\alpha_{q}>0$, Lemma 2 implies that $a_{[q]+1}, a_{[2 \sigma]+1}$, $\cdots, \alpha_{[(q-1) G]+1}$ are $>a_{[q q]+1}$. Also $a_{[q G]+1}=([q G]+1) n_{1}-q d$ and so is $>0$. Now for each $t, 1 \leqq t \leqq q, a_{[t \theta]}>0$ for otherwise $t d$ would be divisible by $n_{1}$ and $\alpha_{t}$ would be 0 . Morever,

$$
a_{1}=n_{1}>[G] n_{1}+\left(n_{1}-d\right)=a_{[a]+1} .
$$

Hence by the discussion of the tables of (4.1) with $k=0,1, \cdots, q-1$ we may conclude that 


$$
a_{1}, a_{2}, \cdots, a_{[q \sigma]} \text { are }>a_{[q \sigma]+1}>0 .
$$

In Lemma 1 set $g=[q G]+1$. This means that $\left(a_{g},[g F]\right)$ is primitive. Thus $q$ was one of the $k_{p}$ 's and the theorem is proved.

As a collollary we give a more convenient computational form of the primitive solutions.

Corollary. Let $(x, y)$ be a solution of $x+d y \equiv 0(\bmod n)$ such that $y=[([i G]+1) F]$. Then $y=[F]([i G]+1)+i$ and $x=n_{1}-\left(i d-[i G] n_{1}\right)$.

Proof. Let $n=q_{1} d+n_{1}, 0<n_{1}<d$. Then $q_{1}=[F]$.

$$
([i G]+1) n=q_{1} d([i G]+1)+n_{1}([i G]+1),
$$

and

$$
y=q_{1}([i G]+1)+\left[G^{-1}([i G]+1)\right] .
$$

Since $i d<n_{1}([i G]+1) \leqq i d+n_{1}<(i+1) d$, we have $y=q_{1}([i G]+1)+i$.

Now write $x=([i G]+1) n-y d$. By substituting $[F]([i G]+1)+i$ for $y$ we obtain $x=n[i G]+n-d[F][i G]-d[F]-i d$. Then substituting $[F] d+n_{1}$ for $n$ and clearing gives $x=n_{1}-\left(i d-[i G] n_{1}\right)$.

In the notation of Theorem 1, let $n>d>n_{1}>d_{1}>0$. Again let $F=n / d$ and $G=d / n_{1}$. Let

$$
\left(x_{1}, y_{1}\right)=(n-[F] d,[F])=\left(n_{1},[F]\right)
$$

and

$\left(x_{2}, y_{2}\right)=\left(n_{1}-d+[G] n_{1},[F]([G]+1)+1\right)=\left(n_{1}-d_{1},[F]([G]+1)+1\right)$.

By Theorem 1 and Corollary, $\left(x_{1}, y_{1}\right)$ and $\left(x_{2}, y_{2}\right)$ are primitive solutions of $x+d y \equiv 0(\bmod n)$. Concerning them we shall now prove the following useful theorem:

THEOREM 2. If $\left(x^{\prime}, y^{\prime}\right)$ is a solution of $x+d y \equiv 0(\bmod n)$ and $(0,0)<\left(x^{\prime}, y^{\prime}\right)<\left(x_{1}+x_{2}, y_{1}+y_{2}\right)$, where $\left(x_{1}, y_{1}\right)$ and $\left(x_{2}, y_{2}\right)$ are the above mentioned primitive solutions, then either $\left(x^{\prime}, y^{\prime}\right)=\left(x_{1}, y_{1}\right)$ or $\left(x^{\prime}, y^{\prime}\right)=$ $\left(x_{2}, y_{2}\right)$

Proof. To prove the theorem we need the following lemma.

LEMma. Suppose there exist primitive solutions $\left(x_{1}, y_{1}\right)$ and $\left(x_{2}, y_{2}\right)$ of $x+d y \equiv 0(\bmod n)$ such that $x_{1}+x_{2} \leqq n, y_{1}+y_{2} \leqq n$ and there is no other primitive solution $\left(x^{\prime}, y^{\prime}\right)$ for which $\left(x^{\prime}, y^{\prime}\right)<\left(x_{1}+x_{2}, y_{1}+y_{2}\right)$. Then there is no other solution $\left(x^{*}, y^{*}\right)$ such that $(0,0)<\left(x^{*}, y^{*}\right)<$ $\left(x_{1}+x_{2}, y_{1}+y_{2}\right)$. 
Proof of lemma. Let $(0,0)<\left(x^{*}, y^{*}\right)<\left(x_{1}+x_{2}, y_{1}+y_{2}\right)$ where $\left(x^{*}, y^{*}\right)$ is a solution of $x+d y \equiv 0(\bmod n)$. Clearly $y^{*} \neq 0$ since $x^{*}$ must be less than $n$. If there are solutions $\left(x^{*}, y^{*}\right)$ with $x^{*}=0$ and $(0,0)<$ $\left(x^{*}, y^{*}\right)<\left(x_{1}+x_{2}, y_{1}+y_{2}\right)$ then select the one with $y^{*}$ minimal. Then $\left(x_{1}+x_{2}, y_{1}+y_{2}-y^{*}\right)$ is a solution and by primitivity $y^{*}>y_{1}, y_{2}$. This implies $y_{1}+y_{2}-y^{*}<y^{*}, y_{1}, y_{2}$. But now the choice of $y^{*}$ implies that there must be a primitive solution $\left(x^{\prime}, y^{\prime}\right) \leqq\left(x_{1}+x_{2}, y_{1}+y_{2}-y^{*}\right)$. Such a primitive solution cannot be $\left(x_{1}, y_{1}\right)$ or $\left(x_{2}, y_{2}\right)$, and this contradicts the hypothesis. Hence we have shown that an arbitrary solution $\left(x^{*}, y^{*}\right)$ which satisfies $(0,0)<\left(x^{*}, y^{*}\right)<\left(x_{1}+x_{2}, y_{1}+y_{2}\right)$ can have neither $x^{*}=0$ nor $y^{*}=0$.

Thus the solution $\left(x^{*}, y^{*}\right)$ must contain a primitive solution. This means for $i=1$ or $2, x_{1} \leqq x^{*}$ and $y_{i} \leqq y^{*}$. For this $i,\left(x^{*}-x_{i}, y^{*}-y_{i}\right)$ is a solution. Either both $x^{*}-x_{i}$ and $y^{*}-y_{i}$ are zero or neither is zero. If $x^{*} \neq x_{i}$ and $y^{*} \neq y_{i}$ then for $j=1$ or $2, x^{*}-x_{i} \geqq x_{j}$ and $y^{*}-y_{i} \geqq y_{j}$. Again, either both $x^{*}-x_{i}-x_{j}$ and $y^{*}-y_{i}-y_{j}$ are zero or neither is zero. Continuing, we obtain

$$
x^{*}=c_{1} x_{1}+c_{2} x_{2} \text { and } y^{*}=c_{1} y_{1}+c_{2} y_{2},
$$

where $c_{1}$ and $c_{2}$ are non-negative integers. If $\left(x_{1}, y_{1}\right)=\left(x_{2}, y_{2}\right)$ then $x^{*}=c x_{1}, y^{*}=c y_{1}$ where $c=c_{1}+c_{2}$. In this case $c$ would be 1 or 2 and the lemma follows. If $\left(x_{1}, y_{1}\right) \neq\left(x_{2}, y_{2}\right)$ we may let $x_{1}>x_{2}, y_{1}<y_{2}$. Then $x_{1}>x_{2}$ and $x^{*} \leqq x_{1}+x_{2}$ imply $c_{1} \leqq 1$ while $y_{1}<y_{2}$ and $y^{*} \leqq y_{1}+y_{2}$ imply $c_{2} \leqq 1$. This proves the lemma.

We must show that the solutions $\left(x_{1}, y_{1}\right)$ and $\left(x_{2}, y_{2}\right)$ of the theorem satisfy the hypothesis of the lemma. One readily verifies that $x_{1}+x_{2}$ and $y_{1}+y_{2}$ are less than $n$. Let $\left(x^{*}, y^{*}\right)$ be a primitive solution such that $(0,0)<\left(x^{*}, y^{*}\right)<\left(x_{1}+x_{2}, y_{1}+y_{2}\right)$. To prove the theorem it suffices to show that $x^{*} \leqq x_{1}$ and $y^{*} \leqq y_{2}$. Now consider the solution $\left(x_{3}, y_{3}\right)$ where

$$
y=[F]([2 G]+1)+2 .
$$

By Theorem 1 and its corollary there is no primitive solution $\left(x^{\prime}, y^{\prime}\right)$ such that $y_{2}<y^{\prime}<y_{3}$. We have

$$
y_{3}-y_{1}-y_{2}=[F]([2 G]-[G]-1)+1 \geqq[F]([G]-1)+1 \geqq 1 .
$$

From this it follows that $y^{*} \leqq y_{2}$.

Now if $[F]=1$, then $x^{*} \leqq x_{1}$. If $[F]>1$, consider the solution $\left(x_{0}, y_{0}\right)=(n-[F] d)+d,[F]-1$. There is no primitive solution $\left(x^{\prime \prime}, y^{\prime \prime}\right)$ such that $x_{0}>x^{\prime \prime}>x_{1}$.

Furthermore

$$
\begin{gathered}
x_{0}-x_{1}-x_{2}=n-[F] d+d-(n-[F] d)-\left(n_{1}-d+[G] n_{1}\right) \\
=2 d-n_{1}-n_{1}[G]>0 .
\end{gathered}
$$


Hence $x^{*} \leqq x_{1}$. Thus $x^{*} \leqq x_{1}$ and $y^{*} \leqq y_{2}$ so that $\left(x^{*}, y^{*}\right)$ equals $\left(x_{1}, y_{1}\right)$ or $\left(x_{2}, y_{2}\right)$. The theorem follows from the lemma.

4. Application. Let $A$ be a cyclic 0,1 matrix of order $n$ defined by differences 0,1 and $d \bmod n$.

As covered in $\S 2$, to show $P(A)>|D(A)|$ it is necessary and sufficient to show the existence of a solution $\left(x^{\prime}, y^{\prime}\right)$ of $x+d y \equiv 0(\bmod n)$ such that $\left(x^{\prime}, y^{\prime}\right)$ represents a cycle and $x^{\prime}+y^{\prime}$ is even. The problem of determining when a solution $\left(x^{\prime}, y^{\prime}\right), 0<x^{\prime}+y^{\prime}$, represents a cycle may be described as follows. Suppose there exists some arrangement of $x^{\prime} \alpha$ 's and $y^{\prime} \beta$ 's in a circle with the following property: For each solution $\left(x^{*}, y^{*}\right)<\left(x^{\prime}, y^{\prime}\right)$, no selection of $x^{*}+y^{*}$ consecutive $\alpha^{\prime}$ 's and $\beta^{\prime}$ 's totals exactly $x^{*} \alpha^{\prime}$ 's (or $y^{*} \beta^{\prime} \mathrm{s}$ ). Then $\left(x^{\prime}, y^{\prime}\right)$ represents a cycle. If no such arrangement is possible then $\left(x^{\prime}, y^{\prime}\right)$ does not represent a cycle.

For the purposes of this section it is not necessary to solve completely this problem in arrangements. In an important class of $d$ and $n$ Theorem 2 yields two primitive solutions $\left(x_{1}, y_{1}\right)$ and $\left(x_{2}, y_{2}\right)$ having the property that there is no other solution $\left(x^{\prime}, y^{\prime}\right)$ such that $(0,0)<\left(x^{\prime}, y^{\prime}\right)<$ $\left(x_{1}+x_{2}, y_{1}+y_{2}\right)$. Thus if there is an arrangement of $x_{1}+x_{2} \alpha$ 's and $y_{1}+y_{2} \beta$ 's in a circle such that no selection of $x_{1}+y_{1}$ consecutive $\alpha$ 's and $\beta$ 's totals exactly $x_{1} \alpha$ 's (or $y_{1} \beta$ 's) then $\left(x_{1}+x_{2}, y_{1}+y_{2}\right)$ represents a cycle. Under these circumstances we have $P(A)>|D(A)|$. For $\left(x_{1}, y_{1}\right)$ and $\left(x_{2}, y_{2}\right)$ represent cycles, and if $x_{1}+y_{1}$ and $x_{2}+y_{2}$ are both odd then $x_{1}+x_{2}+y_{1}+y_{2}$ is even. The proof of Theorem 3 is based upon this device.

THEOREM 3. Let $A$ be a cyclic 0, 1 matrix of order $n$ defined by the differences $0,1, d \bmod n$. Then $P(A)=|D(A)|$ if and only if $n=7$ and $d=3$ or 5 .

Proof. If $n=7$ and $d=3$ or 5 then $P(A)=|D(A)|$ by Example 1. We assume $P(A)=|D(A)|$ and will show $n=7$ and $d=3$ or 5 . If $n$ or $d$ is even then $P(A)>|D(A)|$. For if $n$ is even the permutation $(1,2, \cdots, n)$ is odd. The solution $\left(x^{\prime}, y^{\prime}\right)=(n-d, 1)$ is primitive so if $n$ is odd and $d$ is even then $x^{\prime}+y^{\prime}=n-d+1$ is even. Thus we may assume that both $n$ and $d$ are odd. As before we set $F=n / d$ and $G=d / n_{1}$.

We may also assume that $\left[F^{\prime}\right]=1$.

For if $[F] \geqq 2$ then $d<n / 2$ and $n-d+1>n / 2+1$. Thus $\left[n(n-d+1)^{-1}\right]=1$. Now

$$
A=I+P+P^{a}
$$

where $P$ is a permutation matrix and $P^{n}=I$. Since $A^{T}=I+P^{-1}+P^{-a}$ and $B=P A^{T}=I+P+P^{n+1-a}$, it follows that $P(A)=|D(A)|$ if and 
only if $P(B)=|D(B)|$. Thus if $[F] \geqq 2$, we may study the matrix $B$ with $\left[n(n-d+1)^{-1}\right]=1$. Hence we may assume $[F]=1$. Note that if $n=7, d=3$ then $n-d+1=5$.

We may assume further that in the notation of Theorem 1, $n>d>n_{1}>d_{1} \geqq 1$. For if $d_{1}=0$ then $n_{1}$ is the greatest common divisor of $n$ and $d$. Since $n_{1}=n-d$ and since $n, d$ are odd, $n_{1}$ would have to be both even and odd.

Finally, we may assume $[G]>1$. For if $[G]=1$, consider the primitive solution $\left(x^{\prime}, y^{\prime}\right)=\left(n_{1}-d_{1},[F]([G]+1)+1\right)$. Since $d$ is odd and $d=n_{1}+d_{1}, x^{\prime}+y^{\prime} \equiv n_{1}+d_{1}+1 \equiv 0(\bmod 2)$.

In the remainder of the proof let $\left(x_{1}, y_{1}\right)$ and $\left(x_{2}, y_{2}\right)$ denote the primitive solutions $\left(n_{1}, 1\right)$ and $\left(n_{1}-d_{1},[G]+2\right)$ respectively, as in Theorem 2.

The proof will be completed by showing that if $n$ and $d$ are not 7 and 5 respectively, then $\left(x_{1}+x_{2}, y_{1}+y_{2}\right)$ represents a cycle. Several cases will be considered.

Case $1 a \quad y_{1}+y_{2} \equiv 0\left(\bmod x_{1}+x_{2}\right)$.

Consider the following circular arrangement of $x_{1}+x_{2} \alpha$ 's and $y_{1}+y_{2} \beta$ 's, where $r$ denotes the quotient $\left(y_{1}+y_{2}\right) /\left(x_{1}+x_{2}\right)$.

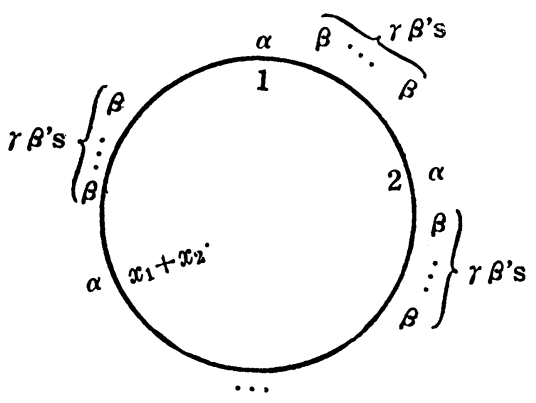

If a selection of $t=x_{1}+y_{1}$ consecutive $\alpha$ 's and $\beta$ 's totals $x_{1} \alpha$ 's then it totals at least $\left(x_{1}-1\right) r \beta$ 's. Let $s$ represent $\left(2 n_{1}-d_{1}\right)^{-1}$. Since $[G] \geqq 2$,

$$
\left(x_{1}-1\right) r=\left(n_{1}-1\right) s([G]+3) \geqq 5 s\left(n_{1}-1\right)>1=y_{1} .
$$

Thus for case $P(A)>|D(A)|$.

Case $1 b \quad y_{1}+y_{2} \not \equiv 0\left(\bmod x_{1}+x_{2}\right)$ and $x_{1}+x_{2}<y_{1}+y_{2}$. Consider the following circular arrangement of $x_{1}+x_{2} \alpha$ 's and $y_{1}+y_{2} \beta$ 's. To simplify the notation we set $u=\left[\left(y_{1}+y_{2}\right) /\left(x_{1}+x_{2}\right)\right]$.

Since $y_{1}+y_{2}-1 \geqq\left(x_{1}+x_{2}\right) u$, we have $y_{1}+y_{2}-1-\left(x_{1}+x_{2}-1\right) u>u$. Thus if $1 \leqq t \leqq x_{1}+x_{2}-1$, a selection of consecutive $\alpha$ 's and $\beta$ 's which totals $t \alpha$ 's will total at most $y_{1}+y_{2}-1-\left(x_{1}+x_{2}-1\right) u+t u+1=$ $y_{1}+y_{2}-\left(x_{1}+x_{2}-t-1\right) u \beta$ 's. 


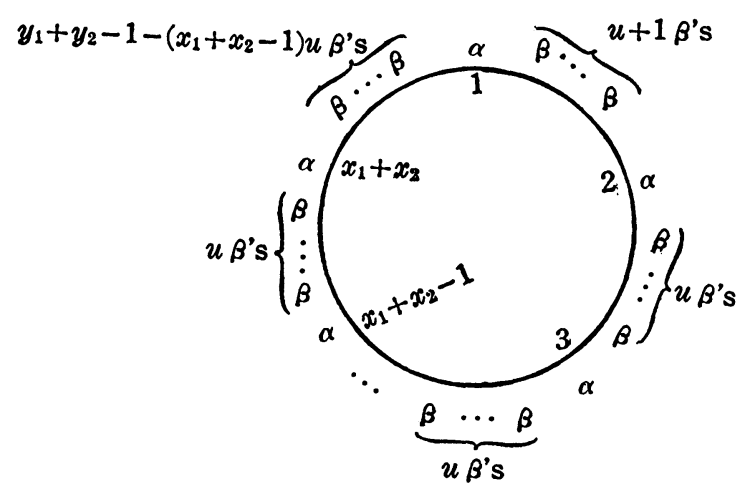

Let $t=x_{2}$ and set $s=\left(2 n_{1}-d_{1}\right)^{-1}$. It suffices to show that $y_{2}>y_{1}+y_{2}-\left(x_{1}+x_{2}-x_{2}-1\right) u$ and thus to show $\left(n_{1}-1\right)[([G]+3) s]>1$. This will be true if $n_{1}>2$.

If $n_{1}=2$ then $d_{1}=1$ and $\left(n_{1}-1\right)[([G]+3) s]=[1 / 3([G]+3)]$. This is $>1$ unless $[G]=2$. But then $d_{1}=1, n_{1}=2=[G]$, and $[F]=1$ together imply $d=5$ and $n=7$.

Case $2 \quad x_{1}+x_{2} \geqq y_{1}+y_{2}$. Consider the following circular arrangement of $\alpha$ 's and $\beta$ 's.

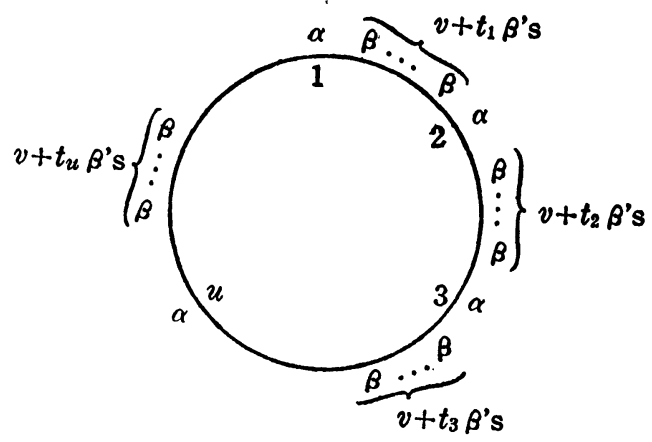

We are supposing throughout the proof that $\left(x_{1}, y_{1}\right)=\left(n_{1}, 1\right)$ and

$$
\left(x_{2}, y_{2}\right)=\left(n_{1}-d_{1},[G]+2\right) \text {. }
$$

Let

$$
u=y_{1}+y_{2}, v=\left[\frac{x_{1}+x_{2}}{y_{1}+y_{2}}\right]
$$

and set $t_{1}=t_{3}=t_{5}=\cdots=0$. For each $i=1,2, \cdots,[u / 2]$ we wish to select $t_{2 i}$ so that $0 \leqq t_{2 i} \leqq n_{1}-2 v-1$ and $t_{2}+t_{4}+t_{6}+\cdots=x_{1}+x_{2}-\left(y_{1}+y_{2}\right) v$. For then any selection of consecutive $\alpha$ 's and $\beta$ 's which totals $y_{1}=1 \alpha$ will total less than $n_{1}=x_{1} \beta$ 's. 
To show that the $t$ 's may be so selected it suffices to prove that

$$
u v+\left[\frac{u}{2}\right]\left(n_{1}-2 v-1\right) \geqq x_{1}+x_{2} .
$$

Let $q=([G]+3)$. We must show that

$$
q\left[\left(2 n_{1}-d_{1}\right) q^{-1}\right]+\left[\frac{1}{2} q\right]\left(n_{1}-2\left[\left(2 n_{1}-d_{1}\right) q^{-1}\right]-1\right) \geqq 2 n_{1}-d_{1} .
$$

The left side equals

$$
\left[\frac{1}{2} q\right] n_{1}+\left[\left(2 n_{1}-d_{1}\right) q^{-1}\right]\left(q-2\left[\frac{1}{2} q\right]\right)-\left[\frac{1}{2} q\right],
$$

and is $\geqq[(1 / 2) q]\left(n_{1}-1\right)$. This is $\geqq 2 n_{1}-d_{1}$ unless $d_{1}=1$ and $[G]=2$. If $d_{1}=1$ and $[G]=2$, then (5.1) becomes

$$
2 n_{1}+\left[\frac{2 n_{1}-1}{5}\right]-2 \text {. }
$$

However,

$$
2 n_{1}+\left[\frac{2 n_{1}-1}{5}\right]-2<2 n_{1}-1
$$

and $n_{1}>1$ together imply that $n_{1}=2$ and hence that $\mathrm{d}=5, n=7$. Thus $(5.1) \geqq 2 n_{1}-d_{1}$ unless $d=5$ and $n=7$.

5. The Main Theorem. In this section we shall obtain a generalization of Theorem 3 by means of elementary group theory. Let $a, b$ be elements of a multiplicative group $G$.

A word is by definition either void and written 1 or a succession $c_{1} c_{2} \cdots c_{q}$ where $c_{i}(i=1,2, \cdots, q)$ is $a$ or $b$. Two words are equal provided they are identical termwise. If $W_{1}=c_{1} c_{2} \cdots c_{r}$ and $W_{2}=$ $c_{r+1} c_{r+2} \cdots c_{r+s}$ are words then the product word $W_{1} W_{2}$ is defined as $c_{1} c_{2} \cdots c_{r} c_{r+1} \cdots c_{r+s}$. If $W_{0}$ is the void word and $W$ is any word then by definition $W_{0} W=W W_{0}=W$. A non-void word $W=c_{1} c_{2} \cdots c_{t}$ will be called a relation between $a$ and $b$ if $c_{1} c_{2} \cdots c_{t}$, considered as an element of $G$, is the identity 1 . The relation will be said to have length $t$. Finally, $W$ is a minimal relation provided $W$ is a relation and any expression of $W$ as a product $W=W_{1} W_{2} W_{3}$ with $W_{2}$ a relation implies that $W=W_{2}$.

TheOREM 4. Let $G$ be a finite group generated by two distinct elements $a$ and $b$. Suppose $H$ is a normal subgroup of $G$ and in the homomorphism $G \rightarrow G / H, a \rightarrow \bar{a}$ and $b \rightarrow \bar{b}$. If there is a minimal relation between $\bar{a}$ and $\bar{b}$ in $G / H$ having even length then there is a minimal relation between $a$ and $b$ in $G$ having even length. 
Proof. Let $\bar{W}$ be a minimal relation between $\bar{a}$ and $\bar{b}$ in $G / H$ having even length. Replace each $\bar{a}$ and $\bar{b}$ in $\bar{W}$ by $a$ and $b$ respectively, thus obtaining a word $W$ on $a$ and $b$. Then $W$ regarded as a group element is in $H$. We may suppose that for some $u>1$, the word $W^{u+1}$ can be written in the form $W^{u+1}=W_{1} W_{2} W_{3}$, where $W_{2}$ is a minimal relation, but $W^{u}$ cannot. The theorem will be proved by showing that either $W_{1}=W_{3}=W_{0}$, the void word, or $W=W_{1} W_{3}$ and consequently that $W_{2}$ is a minimal relation of even length.

By the choice of $u$, there are words $W_{1}^{\prime}, W_{3}^{\prime}$ such that

$$
W_{2}=W_{3}^{\prime} W^{u-1} W_{1}^{\prime} \text { and } W=W_{1} W_{3}^{\prime}=W_{1}^{\prime} W_{3} .
$$

If $W_{3}^{\prime} \neq W_{3}$ then either

(1) $W_{3}^{\prime}=W_{3}^{*} W_{3}$ where $W_{3}^{*}$ is a non-void word, or

( 2) $W_{1}=W_{4} W_{5}, W_{3}=W_{5} W_{6}, W=W_{4} W_{5} W_{6}$ where $W_{4}, W_{5}, W_{6}$ are not void. Since the word $W_{2}$ is a relation, both the group elements $W$ and $W_{1} W_{3}$ are in $H$.

Thus if (1), $W=W_{1} W_{3}^{*} W_{3}$ implies the group element $W_{3}^{*}$ is in $H$. This contradicts the minimality of the relation $\bar{W}$ unless $W_{1}=W_{3}=W_{0}$, the void word.

If (2), the group element $W_{5}$ must be in $H$, contradicting the minimality of $\bar{W}$.

With the aid of Theorem 4 the following generalization of Theorem 3 will now be proved:

THEOREM 5. Let $I$ (the identity), $P$ and $Q$ be disjoint permutations on the letters $1,2, \cdots, n$ such that $P Q=Q P$ and let $A$ be the 0,1 matrix of order $n$ defined by them. Suppose the permutation group $G$ generated by $P$ and $Q$ is transitive. Then $P(A)=|D(A)|$ if and only if upon simultaneous permutations of rows and columns $A$ is transformed into the cyclic $7 \times 7$ matrix $C$ defined by differences $0,1,3 \bmod 7$.

Proof. The sufficiency is a consequence of Theorem 3. The necessity will be proved by induction on $n$. If $n=3$ then $P(A)>|D(A)|$ and the theorem is true. Let $B$ be of order $N, 3 \leqq N<n$, and defined by the disjoint permutations $I, P^{\prime} Q^{\prime}$ where the group $G^{\prime}$ generated by $P^{\prime}$ and $Q^{\prime}$ is transitive and abelian. Moreover, let $P(B)=|D(B)|$. Then the induction hypothesis asserts that $B$ is transformable into $C$ by simultaneous permutations of rows and columns.

Since $G$ is abelian, $G$ is regular and of order $n$. Hence $i, i^{P}, i^{P^{2}}, \cdots$, $i^{P^{x_{1}}}, i^{P^{x_{1}}}, \cdots i^{P^{x_{1} Q^{y_{1}}}}, \cdots, i^{P^{x_{1} Q_{1} P^{x_{2}} Q_{2} \cdots}}$ is a cycle if and only if $P^{x_{1}} Q^{y_{1}} P^{x_{2}} Q^{y_{2}} \cdots$ is a minimal relation between $P$ and $Q$. Thus $P(A)=|D(A)|$ if and only if every minimal relation between $P$ and $Q$ has odd length.

If $G$ is not cyclic then $G$ is homomorphic to an elementary $p$ group 
$\bar{G}$ of type $(p, p)$. Under this homomorphism, denote the images of $P$ and $Q$ by $\bar{P}$ and $\bar{Q}$, respectively. Since $\bar{P}$ and $\bar{Q}$ must be independent generators of $\bar{G}$, the relation $(\bar{P} \bar{Q})^{p}$ is minimal. Thus by Theorem 4 there is a minimal relation between $P$ and $Q$ having even length. Hence $P(A)>|D(A)|$.

Now suppose $G$ is cyclic, $P(A)=|D(A)|$ and consider two cases: (1) $G$ is generated by $P$ and (2) Neither $P$ nor $Q$ generate $G$.

Case 1. Since $G$ is transitive $P$ must be a cycle of length $n$. Thus there is a permutation $R$ such that $R^{-1} P R=(1,2, \cdots, n)$. Consider the $n \times n$ cyclic matrix $A^{*}$ defined by $I, R^{-1} P R, R^{-1} Q R$. $\quad A^{*}$ is obtained from $A$ by simultaneous permutations of rows and columns so by Theorem 3 , $A^{*}$ and hence $A$ is transformable into $C$. Note here that the cyclic $7 \times 7$ matrix defined by differences $0,1,5 \bmod 7$ is transformable into $C$.

Case 2. For this case $n$ must be divisible by at least two distinct primes $p_{1}, p_{2}$. We show first that $n=p_{1} p_{2}$. Let $H_{1}, H_{2}$ be subgroups of $G$ having orders $p_{1}$ and $p_{2}$ respectively. If $H_{1}$ does not contain any of $P, Q, P Q^{-1}$ consider the homomorphism $G \rightarrow G^{\prime}$ where $G^{\prime}$ is the regular representation of $G / H_{1}$ Let $P \rightarrow P^{\prime}, Q \rightarrow Q^{\prime}$ and form the $n / p_{1} \times n / p_{1}$ matrix $A^{\prime}$ defined by $I, P^{\prime}$ and $Q^{\prime}$. The group $G^{\prime}$ is generated by $P^{\prime}, Q^{\prime}$ and is cyclic and transitive. Moreover, $P^{\prime}, Q^{\prime}$ and $I$ are disjoint permutations. By Theorem 4 every minimal relation in $G^{\prime}$ between $P^{\prime}$ and $Q^{\prime}$ must have odd length. Thus $P\left(A^{\prime}\right)=\left|D\left(A^{\prime}\right)\right|$ and, by the induction hypothesis, $A^{\prime}$ is transformable into $C$. Hence $p_{2}=7$ and $n=p_{1} p_{2}$.

If both $H_{1}$ and $H_{2}$ contain one of the three elements $P, Q, P Q^{-1}$ then since $P$ and $Q$ generate $G, G=H_{1} \times H_{2}$. Hence again $n=p_{1} p_{2}$.

We may thus suppose that $P$ has order $p_{1}$ and $Q$ has order $p_{2}$. Now consider the $n \times n$ matrix $A^{*}$ difined by $I, Q^{-1}, P Q^{-1}$.

Since $P(A)=|D(A)|$ we have $P\left(A^{*}\right)=\left|D\left(A^{*}\right)\right|$. However $Q^{-1}$ and $P Q^{-1}$ generate $G$ and $P Q^{-1}$ has order $n$. By Case 1 then $n$ must be 7 so that Case 2 does not arise.

CoROllary 1. Let $I, P, Q$ be disjoint permutations on the letters $1,2, \cdots, n$ such that $P Q=Q P$ and let $A$ be the 0,1 matrix of order $n$ defined by them. Then $P(A)=|D(A)|$ if and only if $n=7 e$ and upon simultaneous permutations of rows and columns $A$ is transformed into the direct sum of $C$ taken e times.

Proof. By the theorem it is sufficient to prove the necessity for the case that the group $G$ generated by $P$ and $Q$ is intransitive.

Let the letters $1,2, \cdots, n$ be divided into $t>1$ transitivity sets containing $N_{1}, N_{2}, \cdots, N_{t}$ letters, respectively. Then upon simultaneous permutations of rows and columns $A$ is transformed into the direct sum 
of $t$ matrices $A_{1}, A_{2}, \cdots, A_{t}$ such that for $1 \leqq j \leqq t, A_{j}$ is of order $N_{j}$ and defined by disjoint permutations $I, P_{j}, Q_{j}$. Moreover $P_{j} Q_{j}=Q_{j} P_{j}$. Applying the theorem to each $A_{j}(j=1,2, \cdots, t)$ proves the corollary.

Corollary 2. Let $A$ be an abelian matrix of order $n$ with 4 ones in each row and column. Then $P(A)>|D(A)|$.

Proof. Without loss we suppose $A$ is defined by permutations $I$, $P, Q, R$ where $P, Q$ and $R$ comute pairwise.

If $P(A)=|D(A)|$ then Corollary 1 implies that $n=7 e$ and there is a permutation $X$ such that the $n \times n$ matrix defined by $I, X^{-1} P X, X^{-1} Q X$ is the direct sum of $C$ taken $e$ times. Now on the letters $1,2, \cdots, 7$, $X^{-1} P X$ and $X^{-1} Q X$ must be the cycles $(1,2, \ldots, 7)$ and $(1,2, \ldots, 7)^{3}$ since these are the only cycles of $C$ having length 7 . Thus on the letters $1,2, \cdots, 7, X^{-1} R X$ must equal $(1,2, \cdots, 7)^{d}$ for some $d \not \equiv 0,1,3$ $(\bmod 7)$. The matrix formed from the first 7 rows and columns of the transformed $A$ is then cyclic and defined by the differences $0,1,3, d$ mod 7. Furthermore by Theorem $3, d=5$. However the $7 \times 7$ cyclic matrix $B$ defined by differences $1,3,5 \bmod 7$ has $P(B)>|D(B)|$. Thus we cannot have $P(A)=|D(A)|$.

It seems to be a plausible conjecture that in Corollaries 1 and 2 the condition that $A$ be abelian may be omitted. Little progress has been made in proving this but it is hoped that in time the full result will yield.

The final theorem will concern the determination of all cyclic matrices $A$ for which $P(A)=|D(A)|$.

A perfect difference set mod $n$ is by definition a set of integers $d_{1}, d_{2}, \cdots, d_{\kappa}, n-1=k(k-1)$, such that every integer $1,2, \cdots, n-1$ is congruent $\bmod n$ to exactly one of the numbers $d_{i}-d_{j}(1 \leqq i, j \leqq k)$. Difference sets have been studied extensively in connection with cyclic projective planes and designs (2.3) but for present purposes we are interested only in a unique role played by the perfect difference set $\bmod 7$.

It is readily verified that if $A$ is a $7 \times 7$ cyclic matrix with $P(A)=$ $|D(A)|$ then $A$ is defined by a perfect difference set mod 7. Also if $A_{1}$ and $A_{2}$ are defined by perfect difference sets $\bmod 7$ then $A_{1}$ may be changed into $A_{2}$ by permutations of rows and columns.

TheOREM 6. Let $A$ by a cyclic 0,1 matrix of order $n$ defined by the differences $0, d_{1}, d_{2} \bmod n$. Then $P(A)=|D(A)|$ if and only if $n=7 e, d_{1}=e d_{1}^{\prime}, d_{2}=e d_{2}^{\prime}$, where $0, d_{1}^{\prime}, d_{2}^{\prime}$ is a perfect difference set $\bmod 7$.

Proof. The sufficiency is a consequence of the following lemma. 
Lemma. Let $B$ be cyclic of order $n$ and defined by the differences $d_{1}, d_{2}, \cdots, d_{s} \bmod n$. For $e \geqq 1$ a positive integer, let $B_{e}$ be the cyclic matrix of order en defined by the differences $e d_{1}, e d_{2} \cdots, e d_{s} \bmod e n$. Then by simultaneous permutations of rows and columns $B_{e}$ may be transformed into the direct sum of $B$ taken e times.

Proof. Let $B_{t}$ denote the $n \times n$ matrix formed from the intersections of the rows $t, e+t, \cdots,(n-1) e+t$ and columns $t, e+t, \cdots,(n-1) e+t$ of $B_{e}$. Here $t$ is a fixed integer on the interval $1 \leqq t \leqq e$, We prove $B_{t}=B$.

Suppose $1 \leqq i, j \leqq n$. There is a 1 in row $(i-1) e+t$, column $(j-1) e+t$ of $B_{e}$ if and only if for some $k, 1 \leqq k \leqq s$,

$$
(j-1) e+t \equiv(i-1) e+t+e d_{\kappa}(\bmod e n) .
$$

This congruence holds if and only if $j \equiv i+d_{\kappa}(\bmod n)$. Thus for each $t, 1 \leqq t \leqq e, B_{t}=B$. Thus the matrix $B_{e}$ contains $e$ principal minors $B$, and these minors are disjoint from one another. This means that by simultaneous permutations of rows and columns we may write $B_{e}$ in the desired form.

The proof of the necessity is by induction. The theorem is true for $n=3$. Let $B$ be cyclic of order $N(3 \leqq N<n)$ and defined by differences $0, a, b \bmod N$ and suppose that $P(B)=|D(B)|$. Then the induction hypothesis asserts that $N=7 l, a=a^{\prime} l$ and $b=b^{\prime} l$ where $0, a^{\prime}, b^{\prime}$ form a perfect difference set $\bmod 7$.

Now let $P(A)=|D(A)|$ and consider the permutation group $G$ generated by $(1,2, \cdots, n)^{d_{1}}$ and $(1,2, \cdots, n) d^{2}$. If $G$ is transitive then Theorem 6 follows by Theorem 5 . If $G$ is intransitive then $\left(d_{1}, d_{2}, n\right)=$ $t>1$ and we may define a cyclic matrix $B$ of order $n / t$ by the differences $0, d_{1} / t, d_{2} / t \bmod n / t$. By the lemma, $P(B)=|D(B)|$. The induction completes the proof.

\section{BIBLIOGRAPHY}

1. König, Dénes, Theorie der Endlichen und Unendlichen Graphen, Chelsea, New York, (1950), 170-178.

2. Hall, Jr. Marshall, Cyclic projective planes, Duke Math. J. 14 (1947), 1079-1090.

3. Hall Marshall and H. J. Ryser, Cyclic incidence matrices, Can. Jour. Math., 3 (1951), 495-502.

The Ohio State University,

Florida State university 


\section{PACIFIC JOURNAL OF MATHEMATICS}

\section{EDITORS}

\author{
David Gilbarg \\ Stanford University \\ Stanford, California \\ F. H. Brownell \\ University of Washington \\ Seattle 5 , Washington
}

\author{
A. L. Whiteman \\ University of Southern California \\ Los Angeles 7, California \\ L. J. PAIGE \\ University of California \\ Los Angeles 24, California
}

\section{ASSOCIATE EDITORS}

\author{
E. F. BECKENBACH \\ T. M. CHERRY \\ D. DERRY
}
E. HEWITT
A. HORN
L. NACHBIN
M. OHTSUKA
H. L. ROYDEN
M. M. SCHIFFER

E. SPANIER

E. G. STRAUS

F. WOLF

\section{SUPPORTING INSTITUTIONS}

\author{
UNIVERSITY OF BRITISH COLUMBIA \\ CALIFORNIA INSTITUTE OF TECHNOLOGY \\ UNIVERSITY OF CALIFORNIA \\ MONTANA STATE UNIVERSITY \\ UNIVERSITY OF NEVADA \\ NEW MEXICO STATE UNIVERSITY \\ OREGON STATE COLLEGE \\ UNIVERSITY OF OREGON \\ OSAKA UNIVERSITY \\ UNIVERSITY OF SOUTHERN CALIFORNIA
}

\author{
STANFORD UNIVERSITY \\ UNIVERSITY OF TOKYO \\ UNIVERSITY OF UTAH \\ WASHINGTON STATE COLLEGE \\ UNIVERSITY OF WASHINGTON

AMERICAN MATHEMATICAL SOCIETY
CALIFORNIA RESEARCH CORPORATION
HUGHES AIRCRAFT COMPANY
SPACE TECHNOLOGY LABORATORIES
NAVAL ORDNANCE TEST STATION

Mathematical papers intended for publication in the Pacific Journal of Mathematics should be typewritten (double spaced), and the author should keep a complete copy. Manuscripts may be sent to any one of the four editors. All other communications to the editors should be addressed to the managing editor, L. J. Paige at the University of California, Los Angeles 24, California. ..

50 reprints per author of each article are furnished free of charge; additional copies may be obtained at cost in multiples of 50 .

The Pacific Journal of Mathematics is published quarterly, in March, June, September, and December. The price per volume (4 numbers) is $\$ 12.00$; single issues, $\$ 3.50$. Back numbers are available. Special price to individual faculty members of supporting institutions and to individual members of the American Mathematical Society: $\$ 4.00$ per volume; single issues, $\$ 1.25$.

Subscriptions, orders for back numbers, and changes of address should be sent to Pacific Journal of Mathematics, 2120 Oxford Street, Berkeley 4, California.

Printed at Kokusai Bunken Insatsusha (International Academic Printing Co., Ltd.), No. $6_{4}$ 2-chome, Fujimi-cho, Chiyoda-ku, Tokyo, Japan.

PUBLISHED BY PACIFIC JOURNAL OF MATHEMATICS, A NON-PROFIT CORPORATION

The Supporting Institutions listed above contribute to the cost of publication of this Journăl, but they are not owners or publishers and have no responsibility for its content or policies. 


\section{Pacific Journal of Mathematics}

\section{Vol. 10, No. $3 \quad$ November, 1960}

Glen Earl Baxter, An analytic problem whose solution follows from a simple

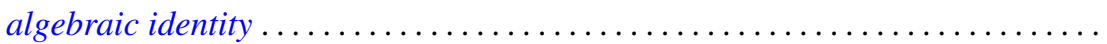

Leonard D. Berkovitz and Melvin Dresher, A multimove infinite game with linear payoff. .

Earl Robert Berkson, Sequel to a paper of A. E. Taylor ......................

Gerald Berman and Robert Jerome Silverman, Embedding of algebraic systems.... 767

Peter Crawley, Lattices whose congruences form a boolean algebra . . . . . ...... 777

Robert E. Edwards, Integral bases in inductive limit spaces . . . . . . . . . . . . . . .

Daniel T. Finkbeiner, II, Irreducible congruence relations on lattices . . . . . . . . . .

William James Firey, Isoperimetric ratios of Reuleaux polygons . . . . . . . . . . . 787

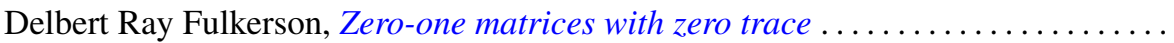

Leon W. Green, A sphere characterization related to Blaschke's conjecture........

Israel (Yitzchak) Nathan Herstein and Erwin Kleinfeld, Lie mappings in

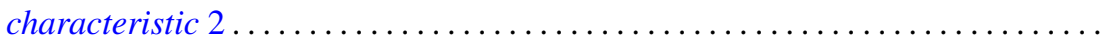

Charles Ray Hobby, A characteristic subgroup of a $p$-group .................

R. K. Juberg, On the Dirichlet problem for certain higher order parabolic

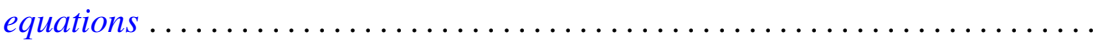

Melvin Katz, Infinitely repeatable games ......................

Emma Lehmer, On Jacobi functions . . . . . . . . . . . . . . . . . . . . . . . . .

D. H. Lehmer, Power character matrices

Henry B. Mann, A refinement of the fundamental theorem on the density of the sum of two sets of integers.

Marvin David Marcus and Roy Westwick, Linear maps on skew symmetric matrices: the invariance of elementary symmetric functions . .

Richard Dean Mayer and Richard Scott Pierce, Boolean algebras with ordered

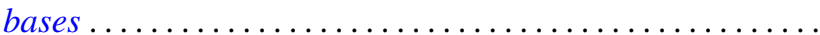

Trevor James McMinn, On the line segments of a convex surface in $E_{3} \ldots$

Frank Albert Raymond, The end point compactification of manifolds ..

Edgar Reich and S. E. Warschawski, On canonical conformal maps of regions of arbitrary connectivity

Marvin Rosenblum, The absolute continuity of Toeplitz's matrices...

Lee Albert Rubel, Maximal means and Tauberian theorems . .

Helmut Heinrich Schaefer, Some spectral properties of positive linear operators

Jeremiah Milton Stark, Minimum problems in the theory of pseudo-conformal transformations and their application to estimation of the curvature of the invariant metric.

Robert Steinberg, The simplicity of certain groups ...

Hisahiro Tamano, On paracompactness. .

Angus E. Taylor, Mittag-Leffler expansions and spectral theory .

Marion Franklin Tinsley, Permanents of cyclic matrices ...... . 